\title{
Coherent structures in the breakdown bubble of a
}

\section{vortex flow}

\author{
Oliver Paterson and Bofu Wang ${ }^{1}$ and Xuerui Mao \\ School of Engineering and Computer Sciences, DurhamUniversity, Durham, DH13LE, UK
}

Helical structures downstream of the breakdown-bubble of the Grabowski vortex profile at Reynolds numbers $R e \leq 500$ have been extensively studied through stability analysis in the literature. However, the low-frequency coherent structures inside the bubble, which have been observed in experiments, were not predicted by the stability approach. In the present work, asymptotic stabilities of the Grabowski vortex flow at $R e \leq 1000$ are carried out to compare against previous works, and then the linear optimal inflow perturbation and the corresponding outcome are studied to unveil the dynamics inside the bubble. It is found that low frequency inflow perturbations penetrate the bubble and are amplified to coherent structures, while high frequency perturbations are convected around the border of the bubble. These linearised results agree well with previous experimental observations and are validated by direct numerical simulations.

\footnotetext{
${ }^{1}$ bofuwang86@gmail.com
} 


\section{Nomenclature}

(Nomenclature entries should have the units identified)

$\boldsymbol{u}, \boldsymbol{U}, \boldsymbol{u}^{\prime}, \boldsymbol{u}^{*}=$ full, base, perturbation and adjoint velocity vectors, respectively

$p, P, p^{\prime}, p^{*}=$ full, base, perturbation and adjoint pressure terms, respectively

$\boldsymbol{u}_{i}^{\prime} \quad=$ inflow velocity perturbation

$\boldsymbol{u}_{\tau}^{\prime}=$ perturbation velocity at final time $\tau$

$x, r, \theta=$ coordinate in the streamwise, radial and azimuthal directions

$S \quad=$ swirl parameter

$\alpha \quad=$ coflow parameter

Re $\quad=$ Reynolds number

$U_{\infty} \quad=$ free-stream axial velocity

$R \quad=$ vortex core radius

$m \quad=$ azimuthal wavenumber

$K \quad=$ energy growth of the optimal inflow perturbation

$\sigma \quad=$ growth rate (real part) and frequency (imaginary part) of the eigenmode

t $\quad$ time

$\tau=$ final time

$\omega \quad=$ frequency

$f \quad=$ temporal function of the boundary perturbation

$\epsilon \quad=$ positive relaxation factor for $f$

$\Omega \quad=$ computational domain

$\partial \Omega \quad=$ inflow boundary

$\mathcal{P}=$ polynomial order in the spectral element method

\section{Introduction}

Vortex dynamics have been a focus of numerous research in fluid mechanics over the past few decades. One of the primary motivations is the dissipation of trailing vortices shed from the wingtips of an airplane [1]. These vortices induce an extra drag component and detrimental effects, e.g. 
downwash and rolling moments, to the following aircraft [2]. To avoid the potential danger of rolling moments caused by trailing vortices, air traffic control set strict spacing rules to separate aircraft during take-off or landing. Another important area of focus is the leading-edge vortices that produce lift and stability on non-commercial delta wing aircraft; sudden changes to the vortex structure can produce drastic and adverse aerodynamic effects [3].

Trailing vortices have been observed to lose stability and breakdown in theoretical, experimental and numerical studies. A widely used definition for vortex breakdown is "an abrupt change in the (vortex) structure with a very pronounced retardation of flow along the axis and a corresponding divergence of the stream surfaces near the axis" [4]. Vortex breakdown can be broadly classified into two types: bubble breakdown, characterised by a recirculation region of low velocity flow occurring immediately downstream of a stagnation point, and spiral breakdown, featuring helical structures either independently or downstream of the bubble breakdown [6-10]. From a stability point of view, it was found that the flow upstream of the bubble breakdown is, at worst, marginally stable, hence the axisymmetric breakdown bubble cannot be a direct consequence of instability [5].

Numerous experimental studies have investigated the internal structure of the bubble-type breakdown using dye visualisation [9-12]. The authors described a process of fluid exchange through simultaneous filling and emptying at the downstream end of the bubble, and observed helical modes within the axisymmetric bubble. As the pattern of dye is strongly affected by the structure instability of bubble and a very weak symmetry breaking of the recirculation region is sufficient to generate a strong symmetry breaking in the dye pattern $[13,14]$, it can be suspected that the asymmetric dye structure does not demonstrate a real flow feature. However, such coherent structures within the breakdown bubble are also observed in several other experiments as well as in the present direct numerical simulations (DNS) studies. In Laser Doppler Anemometry measurements, it was revealed that the interior region of the bubble is dominated by low-frequency motions $[3,15]$ and there is a clear shift of the dominant frequency from upstream to downstream of the bubble [16]. Most recently using Particle Image Velocimetry, a transient double helix mode was observed in the vortex core upstream and inside the bubble [17].

Among the analytical forms of vortices, the Grabowski profile [18], which is a solution of the 
steady axisymmetric Navier-Stokes (NS) equations, has been widely adopted. This profile has a uniform axial velocity beyond a characteristic radius. Scaling the length with the characteristic core radius and the velocities with the free-stream axial velocity, the azimuthal, radial and streamwise velocity components, denoted as $w, v$ and $u$, can be expressed as

$$
\begin{aligned}
w(0 \leq r \leq 1) & =S r\left(2-r^{2}\right) \\
w(1 \leq r) & =S / r \\
v(r) & =0 \\
u(0 \leq r \leq 1) & =\alpha+(1-\alpha) r^{2}\left(6-8 r+3 r^{2}\right), \\
u(1 \leq r) & =1
\end{aligned}
$$

where $r$ is the non-dimensional radial coordinate. In this profile, $S$ is the swirl parameter, defined as the ratio of azimuthal velocity at the edge of the core to the free-stream axial velocity. The effects of this parameter have been studied in depth $[19,20]$. In the present work, $S=1.095$ is adopted to match the previous numerical setup [19]. The coflow parameter, $\alpha$, represents the axial velocity at the axis relative to the free-stream axial velocity. This study is focused on the region $1<\alpha<2$ where the flow is jet-like $(\alpha>1)$ and not inherently stable $(\alpha>2)$.

Ruith et al. [19] conducted DNS of the Grabowski profile and found that parameter combinations were accurately predicted by Benjamin's theory [21]: onset of vortex breakdown is a finite transition between two states, upstream supercritical flow and downstream subcritical flow. Moreover, these authors showed a large pocket of absolute instability that governs the spiral breakdown mode in the wake of the bubble, and obtained eigenfunctions related to helical and double-helical breakdown modes (azimuthal wavenumber $m=-1$ and $m=-2$, respectively). Some of the findings were confirmed by experimental work of Liang and Maxworthy [22].

By performing local stability analysis on the flow fields obtained by Ruith et al. [19] , Gallaire et al. [23] found two regions of absolute instability for a particular swirl parameter. One region is centred on the bubble and the other in the wake behind it. They interpreted the first absolute instability as a result of presence of counterflow in the bubble, and the second one as a cause of nonlinear global mode which triggers spiral vortex breakdown in the wake of the bubble. Meliga and Gallaire [24] conducted a global linear stability of the axisymmetric vortex and further con- 
firmed that the spiral vortex breakdown is induced by an unstable eigenmode. Meliga et al. [25] subsequently extend their analysis to consider weakly nonlinear mechanism that are responsible for competition between helical and double-helical breakdown. Recently, Qadri et al. [26] performed structure sensitivity analysis based on a low-Mach-number formulation of NS equations using the Grabowski inlet profile. Their analysis showed that the region around the bubble is more influential than the wake in determining the growth rate and frequency.

The above instability analysis explains the helical structures downstream of the breakdown bubble, but is unable to account for the low frequency perturbation within the bubble observed in experiments $[3,15,16]$. In this investigation, both initial and boundary perturbations are applied to reveal that the internal structure of the bubble is induced by the amplification of upstream perturbations.

\section{Methodology}

\section{A. Governing equations}

This study assumes the fluid is Newtonian and incompressible, hence the flow dynamics in the cylindrical frame with $x, r$, and $\theta$ denoting the streamwise, radial and azimuthal coordinates, respectively, are governed by the incompressible NS equations

$$
\partial_{t} \boldsymbol{u}=-(\boldsymbol{u} \cdot \boldsymbol{\nabla}) \boldsymbol{u}-\boldsymbol{\nabla} p+R e^{-1} \nabla^{2} \boldsymbol{u} \quad \text { with } \quad \boldsymbol{\nabla} \cdot \boldsymbol{u}=0
$$

where $\boldsymbol{u}$ denotes the velocity field, $p$ is the pressure, $\partial_{t}$ is the time-derivative, and $R e$ is the Reynolds number defined as

$$
R e=\frac{U_{\infty} R}{\nu}
$$

based on the free-stream velocity $U_{\infty}$, the vortex core radius $R$ and the kinematic viscosity $\nu$.

In the case of linear perturbation analysis, the flow field is decomposed into a base flow and a perturbation, i.e. $\boldsymbol{u}=\boldsymbol{U}+\boldsymbol{u}^{\prime}$. Substitute this decomposition into (2) and neglect the high-order terms of perturbations, the linearised NS (LNS) equations can be obtained:

$$
\partial_{t} \boldsymbol{u}^{\prime}=-\left(\boldsymbol{u}^{\prime} \cdot \boldsymbol{\nabla}\right) \boldsymbol{U}-(\boldsymbol{U} \cdot \boldsymbol{\nabla}) \boldsymbol{u}^{\prime}-\boldsymbol{\nabla} p^{\prime}+R e^{-1} \nabla^{2} \boldsymbol{u}^{\prime} \quad \text { with } \quad \boldsymbol{\nabla} \cdot \boldsymbol{u}^{\prime}=0
$$


The base flow is considered as a two-dimensional, steady solution of the NS equations subject to the inflow conditions described in (1). For such an azimuthally homogeneous base flow, eigenmodes of perturbations with different azimuthal wave numbers are decoupled and can be studied individually. Subsequently, the perturbations can be decomposed in the azimuthal direction and considered in the global eigenmode form:

$$
\left(\boldsymbol{u}^{\prime}, p^{\prime}\right)=\left(\boldsymbol{u}_{m}^{\prime}, p_{m}^{\prime}\right) \mathrm{e}^{(\mathrm{i} m \theta+\sigma t)},
$$

where $m$ is the azimuthal wavenumber, and $\operatorname{Re}(\sigma)$ and $\operatorname{Im}(\sigma)$ are the growth rate and frequency of the perturbation. Hereafter, the term perturbation is referred to as the perturbation at a given azimuthal wavenumber $m$ and the subscript $m$ is omitted.

\section{B. BiGlobal stability analysis}

In the global stability analysis undertaken, the most unstable mode and its growth rate at a given azimuthal wavenumber is calculated. The LNS equations (4) are integrated iteratively to establish a Krylov sequence, from which the leading eigenvalues and eigenmodes can be extracted using an Arnoldi method. Such an Arnoldi approach has been extensively used in stability, transient growth and sensitivity calculations and is not elaborated on here [27, 28].

\section{Optimal inflow boundary perturbations}

To investigate the influence of upstream perturbations to the vortex flow, Dirichlet-type velocity inflow perturbations are studied. When the magnitude of inflow perturbation (relative to the base flow) is small, so is the perturbation it induces inside the domain, and therefore the perturbation field can be governed by the LNS equations.

The dimension of the numerically discretised velocity vector can be reduced by separating the spatial and temporal dependence as

$$
\boldsymbol{u}^{\prime}(r, x, t)=\boldsymbol{u}_{i}^{\prime}(r, x) f(t) \quad \text { where } \quad f(t)=\left(1-\mathrm{e}^{\epsilon t^{2}}\right)\left(1-\mathrm{e}^{-\epsilon(\tau-t)^{2}}\right) \mathrm{e}^{\mathrm{i} \omega t},
$$

where $\epsilon$ is a positive relaxation factor. In this definition, $\boldsymbol{u}_{i}^{\prime}(r, x)$ and $f(t)$ denote the spatial and temporal dependences, respectively. The first two factors in the definition of $f(t)$ are introduced to 
remove the numerical discontinuity, with relaxation factor $\epsilon$ used to limit the influence of these two factors, and $\omega$ acts as the frequency of the boundary perturbation if the final time $\tau$ is large enough. The positive relaxation factor is set to $\epsilon=100$ throughout this work to ensure convergence.

The most energetic inflow perturbation can be defined as the perturbation that induces the maximum gain, denoted as $K$, over time interval $\tau$ :

$$
K=\max _{\boldsymbol{u}_{i}^{\prime}} \frac{\int_{\Omega} \boldsymbol{u}_{\tau}^{\prime} \cdot \boldsymbol{u}_{\tau}^{\prime} \mathrm{d} V}{\int_{\partial \Omega} \boldsymbol{u}_{i}^{\prime} \cdot \boldsymbol{u}_{i}^{\prime} \mathrm{d} s}
$$

where $\Omega$ and $\partial \Omega$ denote the computational domain and the inflow boundary, respectively, and $\boldsymbol{u}_{\tau}^{\prime}$ represents the perturbation at $t=\tau[29]$.

In order to calculate the optimal boundary perturbations, the adjoint equations of the LNS equations (4) must also be introduced:

$$
\partial_{t} \boldsymbol{u}^{*}+\boldsymbol{U} \cdot \boldsymbol{\nabla} \boldsymbol{u}^{*}-\boldsymbol{\nabla} \boldsymbol{U} \cdot \boldsymbol{u}^{*}-\nabla p^{*}+R e^{-1} \nabla^{2} \boldsymbol{u}^{*}=0 \quad \text { with } \quad \boldsymbol{\nabla} \cdot \boldsymbol{u}^{*}=0 .
$$

The LNS equations (4) are used to evolve the inflow velocity perturbation over time $t=0$ to $t=\tau$. The adjoint LNS equations (8) can then be initialised using the final condition and evolved backwards. Similar to the system described in Section II B, a Krylov sequence can be built using the iterative process and the optimal or most energetic inflow perturbation can be extracted using an Arnoldi method, as has been well documented in previous literature [29].

\section{Discretisation and convergence}

Since the base flow is homogeneous in the azimuthal direction, it can be calculated by $2 \mathrm{D}(x-r$ plane) DNS. In global stability and boundary perturbation analysis, perturbations have a specified azimuthal wavenumber and therefore the development can be also computed in the $2 \mathrm{D}$ domain. In each 2D $x-r$ plane, the domain is decomposed into 2223 spectral elements, as shown in Figure 1. In each element, a spectral method with order $\mathcal{P}$ is adopted to further decompose it into a $\mathcal{P} \times \mathcal{P}$ grid. For 3D DNS, a complex Fourier decomposition is applied to discretise the azimuthal direction and 16 Fourier modes are calculated [30].

The growth rate of the most unstable mode at $R e=1000, \alpha=1.2$ and $m=2$ is displayed in Table 1 . The growth rate at $\mathcal{P}=7$ has converged to a relative error of less than $1 \%$ with respect 
(a)

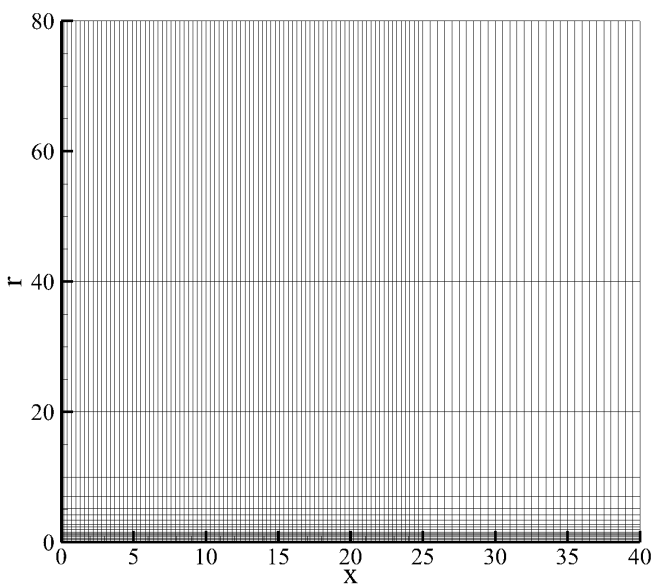

(b)

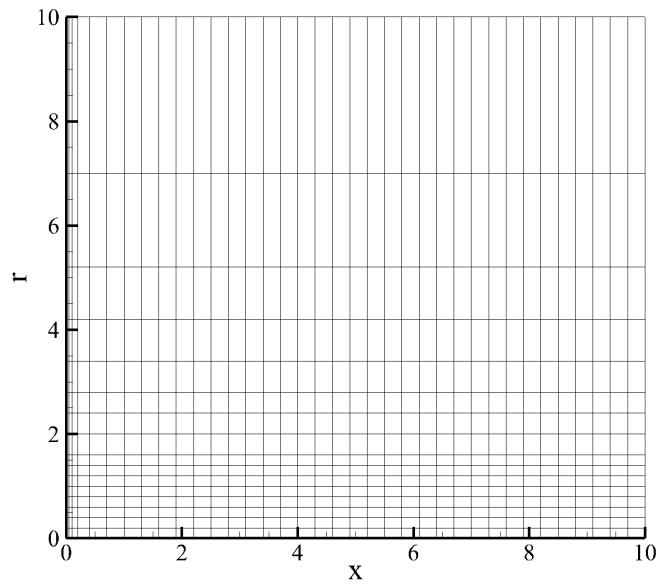

Fig. 1 Discretisation of (a) the overall domain and $(b)$ the subdomain close to inflow boundary and vortex axis, into spectral elements.

\begin{tabular}{ccc}
\hline \hline $\mathcal{P}$ & $\operatorname{Re}(\sigma)$ & \% difference \\
\hline 3 & 0.827200 & 321.632 \\
4 & 0.265930 & 35.547 \\
5 & 0.204930 & 4.455 \\
6 & 0.190980 & 2.656 \\
7 & 0.198040 & 0.943 \\
8 & 0.196190 & - \\
\hline \hline
\end{tabular}

Table 1 Convergence of the growth rate $\operatorname{Re}(\sigma)$ of the most unstable mode with respect to the polynomial order $\mathcal{P}$ in spectral decomposition of each element. Parameters at $R e=1000$, $\alpha=1.2$ and $m=2$.

to $\mathcal{P}=8$. At lower Reynolds number, the relative error is much smaller. Therefore, for cases with $R e=1000, \mathcal{P}=7$ is used to ensure accuracy, whereas at lower Reynolds numbers, $\mathcal{P}=5$ is adopted to reduce computational cost.

\section{Base flow}

Previous work undertaken by Ruith et al. [19] and Grabowski and Berger [18] demonstrated that the base flow at $R e=200$ and $\alpha=1.2$ contained one breakdown bubble, located immediately downstream of the inflow boundary. Ruith et al. [19] further evaluated base flows up to $R e=500$ 
$(a)$

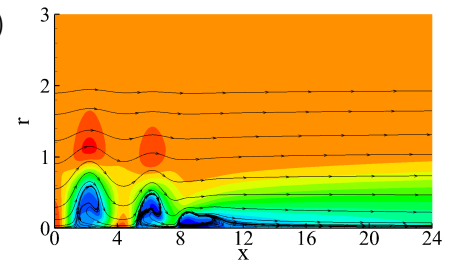

(b)

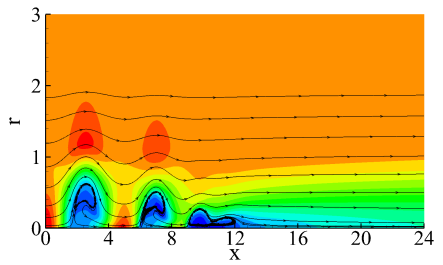

(c)

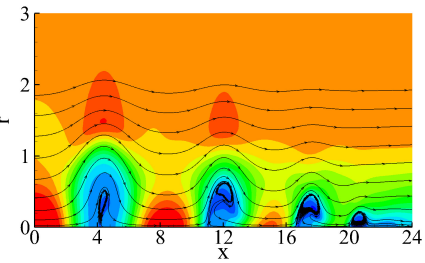

Fig. 2 Contours of streamwise velocity in the $2 \mathrm{D}$ steady base flow at $R e=1000$ and $(a) \alpha=1.2$, (b) $\alpha=1.3$ and (c) $\alpha=1.6$.

with $\alpha=1$, and found two recirculation regions in the highest velocity cases used. For these parameter selections, the current results show good agreement in terms of the location and size of the bubble. It is noted that these Reynolds numbers are still much smaller than that in aeronautical engineering, but the observations can be expected to shed light to the higher Reynolds number cases. To balance the need for real applications and computational cost, the present study is focused on $R e=1000$. To compare against existing works, $R e=200$ and 500 are also considered and briefly discussed.

The 2D flow at $R e=1000$ and various $\alpha$ is displayed in Figure 2. Multiple smaller secondary bubbles are observed trailing the large one immediately downstream of the inflow boundary. In this study, the primary large bubble is referred to as the breakdown bubble and all downstream features as wake structures. The base flow at $\alpha=1.2$ features two recirculation regions in the wake of the primary bubble centred at $x \leq 1.5$. Increasing to $\alpha=1.3$ produces very similar bubbles that form slightly further downstream. At $\alpha=1.6$ the wake structures are elongated, and even though the recirculation region of the primary breakdown bubble is smaller, the deflection of streamlines is larger than that of lower coflow cases.

\section{Global stability analysis}

In this section, stability of the base flow and how it is affected by the evolution of its most unstable mode is investigated. The growth rate of the most unstable modes at three Reynolds numbers of interest, i.e. $R e=200,500$ and 1000, is displayed in Figure 3. Over the parameters considered, the growth rate increases with Reynolds number and maximises at $m=1$; all unstable modes appear at either $m=1$ or $m=2$, a good agreement with previous work [19, 20]. At 
(a)

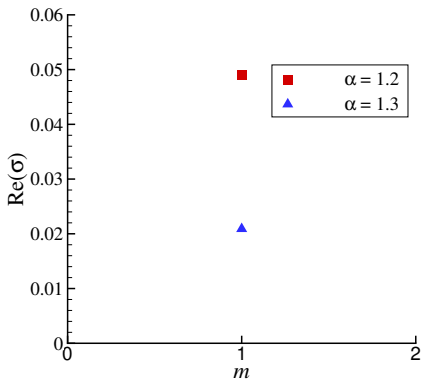

(b)

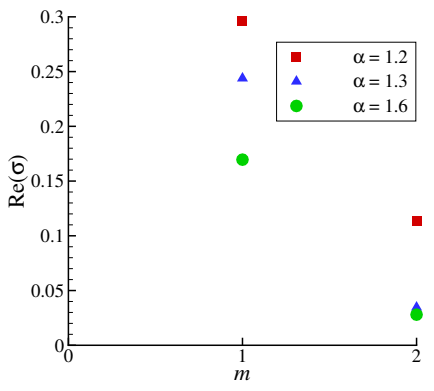

(c)

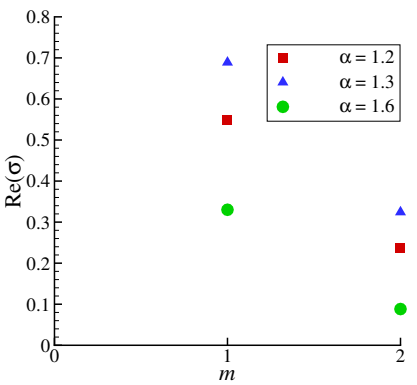

Fig. 3 The growth rate $\operatorname{Re}(\sigma)$ of most unstable modes at Reynolds numbers $(\boldsymbol{a}) \operatorname{Re}=200,(\boldsymbol{b})$ $R e=500$ and $(c) R e=1000$.

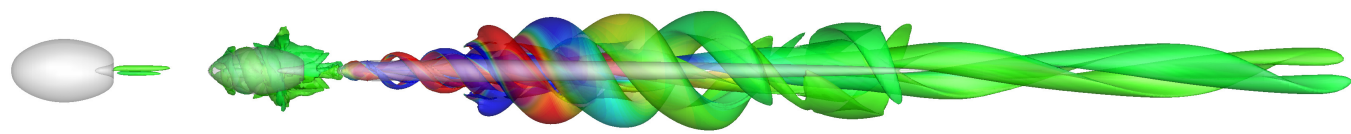

Fig. 4 Iso-surface of $\lambda_{2}$ coloured by streamwise velocity (red for most positive and blue for most negative) for the most unstable mode at $R e=1000, \alpha=1.2$ and $m=1$. The grey bubbles denote the recirculation zones in the base flow.

$R e=1000$, the most unstable mode is found at $\alpha=1.3$, whereas for lower Reynolds numbers, it is at $\alpha=1.2 ; \alpha=1.6$ is consistently the least unstable case. This is reinforced by a previous observation at $R e=200$ that the growth rates reduce when the axial velocity relative to the freestream is increased [19].

The most unstable mode for $R e=1000, \alpha=1.2$ is shown in Figure 4 using the iso-surface of $\lambda_{2}$, whose negative value can be used to identify a vortex [31]. It is clear that the most unstable mode takes the form of helical structures and is concentrated in the wake of the primary bubble of the base flow. The frequency of this mode is $\omega=1.2$, which will be denoted as the instability frequency in the following. Qualitatively, cases at $\alpha=1.3$ and 1.6 display very similar results where the significant region of the most unstable eigenmode is far downstream from the primary bubble.

Influences of the instability can be studied through non-linear development of the most unstable mode using 3D DNS. The iso-surface of $\lambda_{2}=-1$ of the DNS result at $R e=1000, \alpha=1.2$ and $m=1$ is displayed in Figure 5. In this figure the flow can be separated into two regions: the upstream axisymmetric breakdown and the downstream helical structures, with a dominant wavenumber 


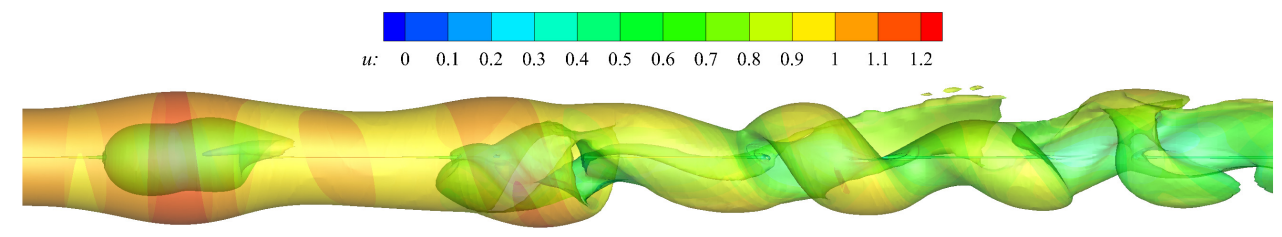

Fig. 5 Iso-surface of $\lambda_{2}=-1$ coloured by streamwise velocity at $R e=1000$ and $\alpha=1.2$ obtained by 3D DNS.

(a)

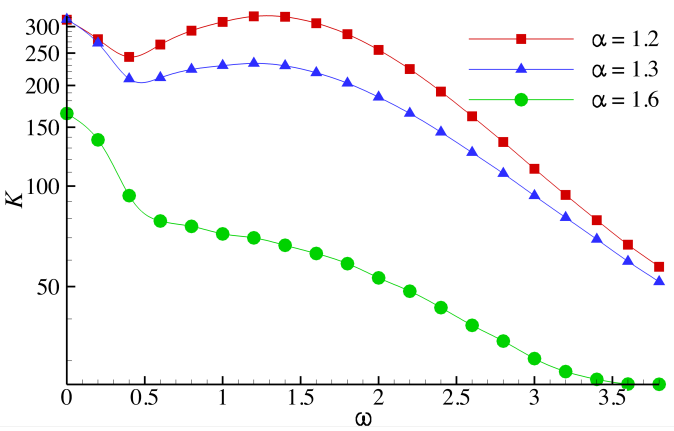

(b)

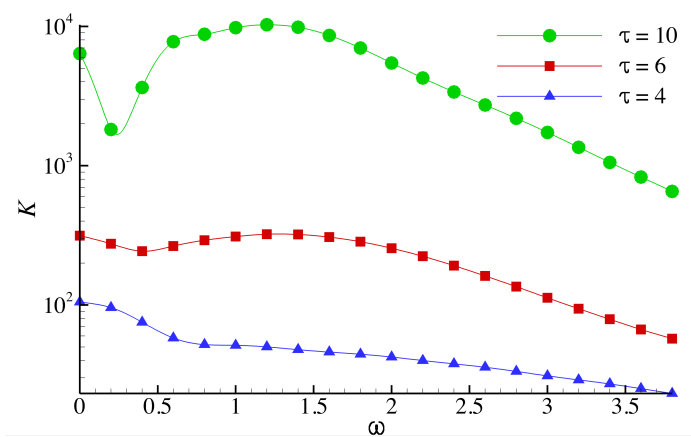

Fig. 6 The gain $K$ of the optimal boundary perturbations at $(a) \tau=6$ and various $\alpha$, and (b) $\alpha=1.2$ and various $\tau$. The Reynolds number and azimuthal wavenumber are $R e=1000$ and $m=1$, respectively.

$m=1$. Therefore, similar to previously reported [20, 23], the global instability analysis in the present work reveals the generation of helical structures downstream of the primary bubble observed in both experiments and simulations. However, such an analysis does not explain the experimentally observed coherent structures inside the primary bubble [15].

\section{Inflow boundary perturbations}

As addressed in the above section, perturbations inside the domain do not induce low-frequency coherent structures in the primary bubble. In this section, the inflow boundary perturbation is considered to model effects of upstream disturbances. Azimuthal wavenumber $m=1$, at which the perturbation growth maximises as shown in Figure 3, is adopted in the following study.

The gain of optimal inflow perturbations is illustrated in Figure 6(a). To calculate the most energetic boundary perturbation that induces largest energy growth inside the bubble, a small final time, $\tau=6$, is adopted. Over the parameters considered, this gain maximises at coflow parameter 
(a)

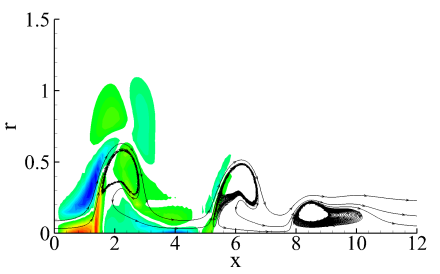

(b)

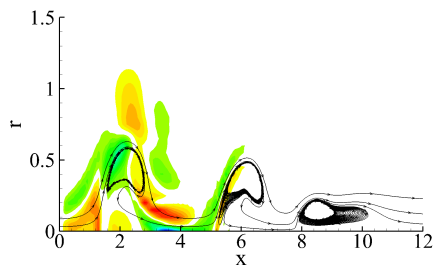

(c)

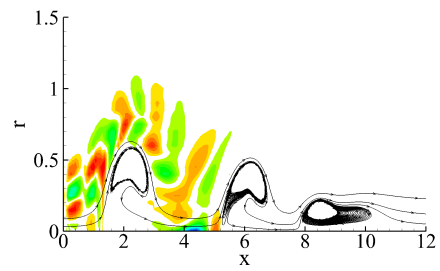

Fig. 7 Contours of azimuthal vorticity (red for most positive and blue for most negative) in the outcomes of the optimal inflow perturbations at $t=6$. The inflow perturbation is obtained at $\tau=6, R e=1000, \alpha=1.2, m=1$ and frequency $(a) \omega=0(b) \omega=1.2(c) \omega=3.8$. $\alpha=1.2$, which will be the focus of the following analysis. Furthermore, the frequency of the inflow perturbation $(\omega)$ has a significant impact on the gain. For $\alpha=1.3$ and 1.6, the largest gain is at $\omega=0$, where $K=315.4$ and 164.9 , respectively. Whereas, the maximum gain for $\alpha=1.2$ is $K=322.2$ at $\omega=1.2$, which agrees with the instability frequency observed in Section V.

Effects of $\tau$ on the gain $K$ is illustrated in Figure $6(b)$, where the coflow parameter is fixed at $\alpha=1.2$. At small values of $\tau$, low-frequency inflow disturbances are more amplified and the gain reduces monotonically with $\omega$. At higher values of $\tau$, the perturbation field is dominated by the global instability downstream of the primary bubble, and therefore, only reiterates the previously well studied downstream helical structures displayed in Section V.

The optimal inflow perturbations obtained at $\tau=6$ and $\alpha=1.2$ are then evolved from $t=0$ to $t=6$ using the LNS equations (4), as illustrated in Figure 7. The inflow perturbation is convected by the base flow and develops spatially and temporally. Therefore, the transient response of the flow to the inflow perturbation is dramatically different with the most unstable mode over the period considered. It is noticed that at frequencies higher than the instability frequency, e.g. $\omega=3.8$, the inflow perturbation is convected around the breakdown bubble, while at low frequencies, the perturbation penetrates into the recirculating bubble region. This frequency dependence is in good agreement with previous experimental studies [3, 15]. Note that this frequency selection behaviour is analogous to shielding effect of boundary layer flow to free-stream noise [32].

The cases with $\alpha=1.3$ and 1.6 display similar mechanisms, with low frequency noise inside the breakdown bubble and high frequency noise convected around. In the case of $\alpha=1.6$, the noise propagates less into the recirculation region due to the smaller size of the axisymmetric bubble; this 


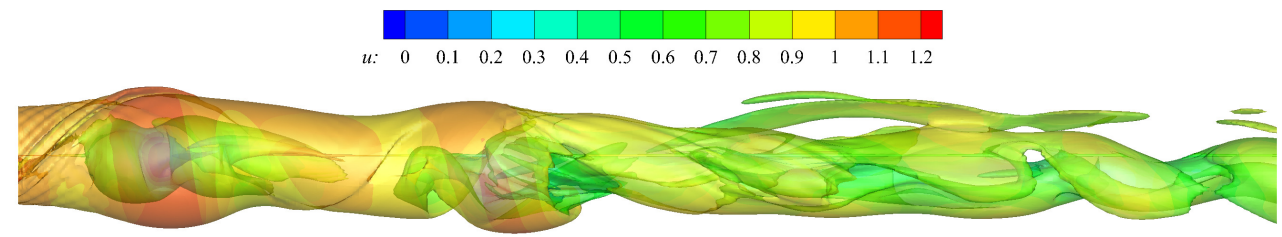

Fig. 8 Iso-surface of $\lambda_{2}=-1$ coloured by streamwise velocity. The flow is calculated through 3D DNS at $R e=1000$ and $\alpha=1.2$, using the Grabowski profile with a random noise as the inflow boundary condition.

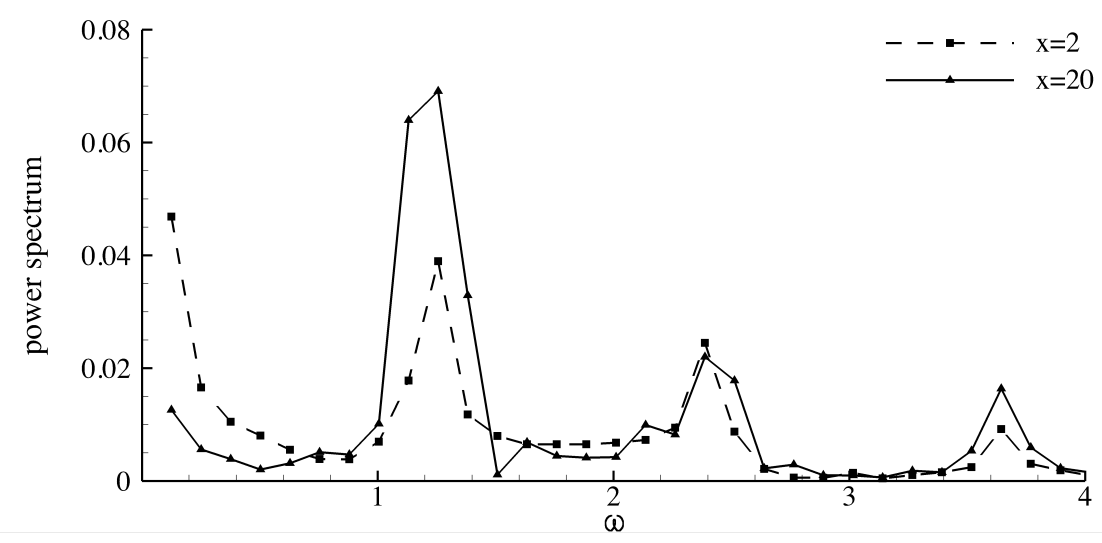

Fig. 9 Power spectrum of the spanwise velocity at $(x, r, \theta)=(2,0.5,0)$ (inside the bubble) and $(20,0.5,0)$ (downstream of the bubble). The other parameters are the same as in Figure 8.

is likely to be the cause of lower gain $(K)$ shown in Figure 6.

To confirm the above linear observations, 3D DNS with a turbulent inflow velocity condition was conducted, as illustrated in Figure 8. It is clear that the axisymmetric bubble breakdown is accompanied by helical noise structures inside, as predicted by the linear study, and the downstream unstable helical instabilities are also activated by the inflow noise. 3D DNS at other coflow parameters were tested (not shown here) and similar structures were observed.

The velocity power spectrum extracted from the 3D DNS is presented in Figure 9. Inside the bubble at $(x, r, \theta)=(2,0.5,0)$, the velocity fluctuation is prominent as the instability frequency $\omega=1.2$ and lower frequencies. The low-frequency fluctuation reflects the transient amplification of inflow disturbance observed in figure 6 . At downstream location $(x, r, \theta)=(20,0.5,0)$, the fluctuation is dominated by the instability frequency and its higher harmonics due to nonlinear effects. 


\section{Conclusion}

A well tested Grabowski profile was adopted in this work to unveil the dynamics in the primary breakdown bubble of a vortex flow. Linear stability, optimal inflow perturbations and DNS were undertaken to track the source of coherent structures inside the bubble, which have been observed in previous experiments but not captured in global stability analysis.

Evaluating the BiGlobal stability allows the flow to be defined as an upstream primary bubble breakdown with a downstream helical mode created by absolute instability within the wake of the bubble, as found by Gallaire et al. [23]. Global stability studies were undertaken at three Reynolds numbers, $R e=200,500$, and 1000. The growth rate increases with Reynolds number, with the most unstable mode always at azimuthal wavenumber $m=1$. The dominant instability mode at $R e=1000, \alpha=1.2$ and $m=1$ has a frequency $\omega=1.2$. All the unstable modes are in the downstream region and have limited effects on the breakdown bubble, in good agreement with previous stability works $[19,23]$.

Through calculating the optimal inflow boundary perturbations amplified over the bubble region, the response of the breakdown bubble to inflow frequency is analysed. At high frequencies (above the instability frequency $\omega=1.2$ ) the perturbation is convected around the bubble and produces low energy growth, whereas low frequency perturbations propagate into the bubble and result in higher energy growth. 3D DNS with a turbulent inflow condition was conducted and the results confirm the linear observations that low-frequency inflow perturbation penetrates into the bubble and develops into coherent structures. This low-frequency structure inside the bubble has previously been examined experimentally [15] but has not been investigated numerically to reveal its mechanism until now.

\section{Acknowledgments}

This work was supported by the Engineering and Physical Sciences Research Council [grant numbers EP/M025039/2]. 


\section{References}

[1] Mager, A., "Dissipation and breakdown of a wing-tip vortex," J. Fluid Mech., Vol. 55, 1972, pp. 609-628, doi:10.1017/S0022112072002046.

[2] Spalart, P. R., "Airplane trailing vortices," Annu. Rev. Fluid Mech., Vol. 30, 1998, pp. 107-38, doi:10.1146/annurev.fluid.30.1.107.

[3] Lucca-Negro, O. and O’Doherty, T., "Vortex breakdown: a review," Prog. Energy Combust. Sci., Vol. 27, 2001, pp. 431-481, doi:10.1016/S0360-1285(00)00022-8.

[4] Hall, M. G., "Vortex breakdown," Annu. Rev. Fluid Mech., Vol. 4, 1972, pp. 195-218, doi:10.1146/annurev.fl.04.010172.001211.

[5] Escudier, M., "Vortex breakdown: observations and explanations," Prog. Aerospace Sci., Vol. 25, 1988, pp. 189-229, doi:10.1016/0376-0421(88)90007-3.

[6] Peckham, D. H. and Atkinson, S. A., "Preliminary Results of Low Speed Wind Tunnel Tests on a Gothic Wing of Aspect Ratio I.0," Tech. rep., Aero. Res. Counc. Tech. Rep. 508, 1960.

[7] Lambourne, N. C. and Bryer, D. W., "Bursting of Leading-Edge Vortices: Some Observations and Discussion of the Phenomenon," Tech. rep., Aero. Res. Counc. R \& M 3282, 1961.

[8] Havery, J. K., "Some observations of the vortex breakdown phenomenon," J. Fluid Mech., Vol. 14, 1962, pp. 585-592, doi:10.1017/S0022112062001470.

[9] Sarpkaya, T., "On stationary and travelling vortex breakdowns," J. Fluid Mech., Vol. 45, 1971, pp. $545-559$, doi:10.1017/S0022112071000181.

[10] Sarpkaya, T., "Vortex Breakdown in Swirling Conical Flows," AIAA Journal, Vol. 9, No. 9, 1971, pp. 1792-1799, doi:10.2514/3.49981.

[11] Sarpkaya, T., "Effect of the Adverse Pressure Gradient on Vortex Breakdown," AIAA Journal, Vol. 12, No. 5, 1974, pp. 602-607, doi:10.2514/3.49305.

[12] Faler, J. H. and Leibovich, S., "Disrupted states of vortex flow and vortex breakdown," Phys. Fluids (1958-1988), Vol. 20, 1977, pp. 1385-1400, doi:10.1063/1.862033. 
[13] Spohn, A., Mory, M., and Hopfinger, E. J., "Experiments on vortex breakdown in a confined flow generated by a rotating disc," J. Fluid Mech., Vol. 370, 1998, pp. 73-99, doi:10.1017/S0022112098002092.

[14] Sotiropoulos, F., Webster, D. R., and Lackey, T. C., "Experiments on Lagrangian transport in steady vortex-breakdown bubbles in a confined swirling flow," J. Fluid Mech., Vol. 466, 2002, pp. 215-248, doi:10.1017/S0022112002001271.

[15] Faler, J. H. and Leibovich, S., "An experimental map of the internal structure of a vortex breakdown," J. Fluid Mech., Vol. 86, 1978, pp. 313-335, doi:10.1017/S0022112078001159.

[16] Garg, A. K. and Leibovich, S., "Spectral characteristics of vortex breakdown flowfields," Phys. Fluids (1958-1988), Vol. 22, No. 11, 1979, pp. 2053-2064, doi: $10.1063 / 1.862514$.

[17] Vanierschot, M., Percin, M., and Oudheusden, B., "Visualization of the structure of vortex breakdown in free swirling jet flow," 18th International Symposium on the Application of Laser and Imaging Techniques to Fluid Mechanics, Lisbon, Portugal.

[18] Grabowski, W. J. and Berger, S. A., "Solutions to the Navier-Stokes equations for vortex breakdown," J. Fluid Mech., Vol. 75, 1976, pp. 525-544, doi:10.1017/S0022112076000360.

[19] Ruith, M. R., Chen, P., Meiburg, E., and Maxworthy, T., "Three-dimensional vortex breakdown in swirling jets and wakes: direct numerical simulation," J. Fluid Mech., Vol. 486, 2003, pp. 331-378, doi:10.1017/S0022112003004749.

[20] Qadri, U. A., Mistry, D., and Juniper, M. P., "Structural sensitivity of spiral vortex breakdown," J. Fluid Mech., Vol. 720, 2013, pp. 558-581, doi:10.1017/jfm.2013.34.

[21] Benjamin, T. B., "Theory of the vortex breakdown phenomenon," J. Fluid Mech., Vol. 14, 1962, pp. 593-629, doi:10.1017/S0022112062001482.

[22] Liang, H. and Maxworthy, T., "An experimental investigation of swirling jets," J. Fluid Mech., Vol. 525, 2005, pp. 115-159, doi:10.1017/S0022112004002629.

[23] Gallaire, F., Ruith, M., Meiburg, E., Chomaz, J., and Huerre, P., "Spiral vortex breakdown as a global mode," J. Fluid Mech., Vol. 549, 2006, pp. 71-80, 
doi:10.1017/S0022112005007834

[24] Meliga, P. and Gallaire, F., "Global instability of helical vortex breakdown," In 6th AIAA Theoretical Fluid Mechanics Conference, 27-30 June 2011, Honolulu, Hawaii.

[25] Meliga, P., Gallaire, F., and Chomaz, J.-M., "A weakly nonlinear mechanism for mode selection in swirling jets," Journal of Fluid Mechanics, Vol. 699, 2012, pp. 216-262.

[26] Qadri, U. A., Mistry, D., and Juniper, M. P., "Structural sensitivity of spiral vortex breakdown," Journal of Fluid Mechanics, Vol. 720, 2013, pp. 558-581.

[27] Schmid, P. J., "Nonmodal Stability Theory," Annu. Rev. Fluid Mech., Vol. 39, 2007, pp. 129-162, doi:10.1146/annurev.fluid.38.050304.092139.

[28] Barkley, D., Blackburn, H. M., and Sherwin, S. J., "Direct Optimal Growth Analysis for Timesteppers," Int. J. Num. Meth. Fluids, Vol. 57, 2008, pp. 1435-1458, doi:10.1002/fld.1824.

[29] Mao, X., Blackburn, H. M., and Sherwin, S. J., "Calculation of global optimal initial and boundary perturbations for the linearised incompressible Navier-Stokes equations," J. Comput. Phys., Vol. 235, 2013, pp. 258-273, doi:10.1016/j.jcp.2012.10.049.

[30] Blackburn, H. M. and Sherwin, S. J., "Formulation of a Galerkin Spectral Element-Fourier Method for Three-Dimensional Incompressible Flows in Cylindrical Geometries,” J. Comput. Phys., Vol. 197, No. 2, 2004, pp. 759-778, doi:10.1016/j.jcp.2004.02.013.

[31] Jeong, J. and Hussain, F., "On the identification of a vortex," J. Fluid Mech., Vol. 285, 1995, pp. 69-94, doi:10.1017/S0022112095000462.

[32] Durbin, P. and Wu, X., "Transition beneath vortical disturbances," Annu. Rev. Fluid Mech., Vol. 39, 2007, pp. 107-128. 